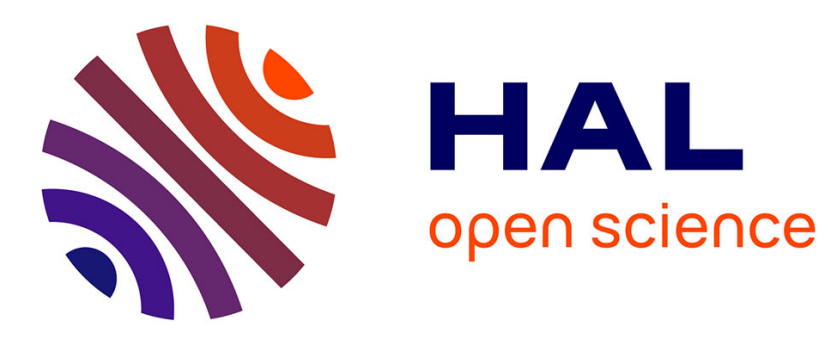

\title{
Air traffic complexity based on dynamical systems
}

Daniel Delahaye, Stéphane Puechmorel

\section{To cite this version:}

Daniel Delahaye, Stéphane Puechmorel. Air traffic complexity based on dynamical systems. CDC 2010, 49th IEEE Conference on Decision and Control, Dec 2010, Atlanta, United States. pp 20692074, 10.1109/CDC.2010.5718004 . hal-00938405

\section{HAL Id: hal-00938405 \\ https://hal-enac.archives-ouvertes.fr/hal-00938405}

Submitted on 5 May 2014

HAL is a multi-disciplinary open access archive for the deposit and dissemination of scientific research documents, whether they are published or not. The documents may come from teaching and research institutions in France or abroad, or from public or private research centers.
L'archive ouverte pluridisciplinaire $\mathbf{H A L}$, est destinée au dépôt et à la diffusion de documents scientifiques de niveau recherche, publiés ou non, émanant des établissements d'enseignement et de recherche français ou étrangers, des laboratoires publics ou privés. 


\title{
Air Traffic Complexity based on Dynamical Systems
}

\author{
Daniel delahaye and Stéphane Puechmorel
}

\begin{abstract}
This paper presents a new air traffic complexity metric based on dynamical systems. Based on a set of radar observations (position and speed) a vector field interpolating these data is constructed. Once the field has been obtained, the Lyapunov spectrum of the associated dynamical system is computed on points evenly spaced on a spatial grid. The results of the computations are summarized on complexity maps, with high values indicating areas to avoid or to carefully monitor. A first approach based on linear dynamical system enable to compute an aggregate complexity metric. In order to produce complexity maps, two extensions of the previous approach have been developed (one in space and another in space and time). Finally, an approximation is proposed in order to localize the computation of the vector field by the mean of Local Linear Models.
\end{abstract}

\section{INTRODUCTION}

The ATM system has to cope with an increasing number of flights, pushing the capacity to its limits. As an example, the average daily traffic above Europe was 26286 flights/day, with a peak traffic demand in excess of 31000 flights [14]. Although delays are kept low, it is expected from the same reference that capacity has to be extended in the future. Basically, two strategies can be devised : adapt the demand to capacity (slot-route allocation, collaborative decision making, ...) or adapt the capacity to the demand (Airspace design, 4D trajectory planning, autonomous aircraft, ...). The first approach can be used in the context of current ATM system, while innovative future designs will mainly follow the second strategy. Currently, complexity of the traffic is measured only as an operational capacity : the maximum number of aircraft that ATC controllers are willing to accept is fixed on a persector basis and complexity is assessed by comparing the real number of aircraft with the sector capacity. It must be noted that under some circumstances, controllers will accept aircraft beyond the capacity threshold while rejecting traffic at other times although the number of aircraft is well below the maximum capacity. This simple fact clearly show that capacity as a crude complexity metric is not enough by itself to fully account for the controller's workload. In order to better quantify the complexity, geometric features of the traffic have to be included. As previously stated, depending on the traffic structure, ATC controllers will perceive differently situations, even if the number of aircraft present in the sector is the same. Furthermore, exogenous parameters like the workload history can be influential on

D. Delahaye is with Applied Math Laboratory, French Civil Aviation University, 7 Avenue Edouard Belin, 31055 Toulouse, France delahayedrecherche.enac.fr

S. Puechmorel is head of Applied Math Laboratory, French Civil Aviation University, 7 Avenue Edouard Belin, 31055 Toulouse, France puechmorerecherche.enac.fr the perceived complexity at a given time (a long period of heavy load will tend to reduce the efficiency of a controller). Some reviews of complexity in ATC have been completed, mainly from the controller's workload point of view [7], [13], and have recognized that complexity is related to both the structure of the traffic and the geometry of the airspace. This tends to prove that controller's workload has two facets :

- An intrinsic complexity related to traffic structure.

- A human factor aspect related to the controller itself.

While most complexity metrics tend to capture those effects within a single aggregate indicator, the purpose of this work is to design a measure of intrinsic complexity only since it is the most relevant metric for an highly automated ATC system (no human factors). The first complexity indicator incorporating structural considerations along with the simple number of aircraft is the "Dynamic Density" of Laudeman et al. from NASA [12]. The "Dynamic Density" is a weighted sum of the traffic density (number of aircraft), the number of heading changes ( $>15$ degrees), the number of speed changes $(>0.02$ Mach), the number of altitude changes ( $>750 \mathrm{ft}$ ), the number of aircraft with 3-D Euclidean distance between 0-25 nautical miles, the number of conflicts predicted in 25-40 nautical miles. These factors are summed together using weighting factors that were determined by showing different traffic scenarios to several controllers. B.Sridhar from NASA [15], has developed a model to predict the evolution of such a metric in the near future. Efforts to define "Dynamic Density" have identified the importance of a wide range of potential complexity factors, including structural considerations. However, the instantaneous position and speeds of the traffic itself does not appear to be enough to describe the total complexity associated with an airspace. A few previous studies have attempted to include structural consideration in complexity metrics, but have done so only to a restricted degree. For example, the Wyndemere Corporation proposed a metric that included a term based on the relationship between aircraft headings and dominant geometric axis in a sector [10]. The importance of including structural consideration has been explicitly identified in work at Eurocontrol. In a study to identify complexity factors using judgment analysis, Airspace Design was identified as the second most important factor behind traffic volume [11]. Histon, et, al. [8], [9] investigated how this structure can be used to support structure-based abstractions that controllers appear to use to simplify traffic situations. The previous models do not take into account the intrinsic traffic disorder which is related to the complexity. The first efforts related with disorder can be found in [4]. This paper introduces two classes of metrics 
which measure the disorder of a traffic pattern.G.Aigoin has extended and refined the geometrical class by using a cluster based analysis [1]. All the previous metrics capture only one feature of the complexity and are not able to produce an aggregate metric which can capture all the possible situations (high-low density, how-low convergence, translation organization, round about organization etc ...). The work presented in this paper is based on dynamical systems modeling of the air traffic. A dynamical system describes the evolution of a given state vector. If such a vector is given by the position of aircraft $\vec{X}=[x, y, z]^{T}$, a dynamical system associates a speed vector $\dot{\vec{X}}=\left[v_{x}, v_{y}, v_{z}\right]^{T}$ to each point in the airspace. The key idea is to find a dynamical system which models the observed aircraft trajectories. Based on this dynamical system modeling, a trajectory disorder metric can be computed.

The first part of this paper will present a linear dynamical system modeling for which the complexity metric can be represented into a complex coordinate system. In this system, it is very easy to identify any speed vector organization pattern. The second part introduces a non linear extension of the previous dynamical system modeling that may model any observed traffic. This extension is done in space only. Such a non linear modeling can be used to produce maps of traffic complexity by identifying areas with high(low) complexity. The third part presents the time extension of the previous model for which works straightly on the trajectory segments instead of speed vectors. Finally, the fourth part, propose an approximation approach based on Local Linear Models for computation improvement. This last model is also able to take into account some uncertainties on aircraft positions in order to improve the robustness of complexity maps.

\section{LinEAR DYNAMICAL SYSTEM MODELING}

The key idea of this approach is to model a set of aircraft trajectories by a linear dynamical system which is defined by the following equation :

$$
\dot{\vec{X}}=\mathbf{A} \cdot \vec{X}+\vec{B}
$$

where $\vec{X}$ is the state vector of the system :

$$
\vec{X}=\left[\begin{array}{lll}
x & y & z
\end{array}\right]^{T}
$$

This equation associates a vector speed $\dot{\vec{X}}$ to a position in the space coordinate $\vec{X}$. The eigenvalues of the matrix $A$ control the evolution of the system. The real part of those eigenvalues is related with the convergence or the divergence property of the system in the direction of the eigenvector. When such a eigenvalue has positive real part, the system is in expansion mode and when it is negative the system is in contraction mode. On the other end, the imaginary part of the eigenvalues are related with the level of curl organization of the system. Depending of those eigenvalues, a dynamical system can evolve in contraction, expansion, rotation or a combination of those three modes. Figure 1 give four typical examples for which the $A$ matrix and the associated eigenvalues are computed. The eigenvalues

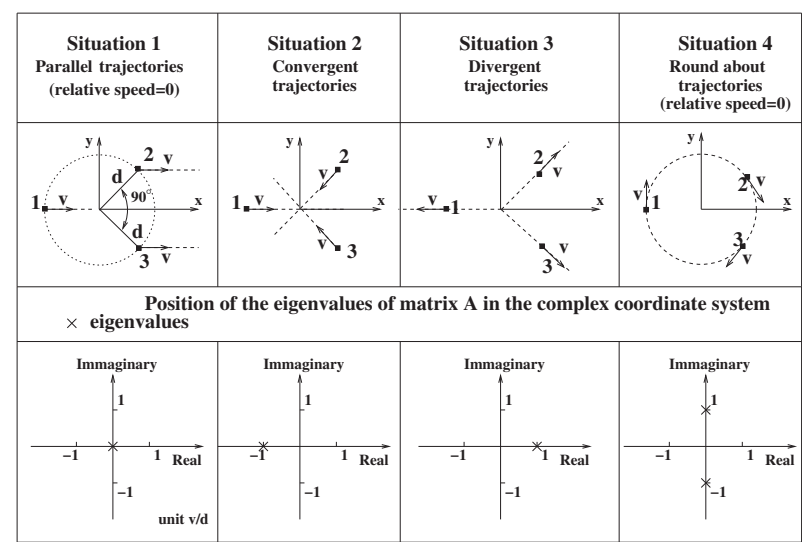

Fig. 1. Eigenvalues loci for several typical situations. The small squares are the initial positions of aircraft at a given time(this represents the observation given by a radar for instance with the associated speed vector).

associated to the matrices $\mathbf{A}$ are given on the bottom part of figure 1 . When the relative distances between aircraft remain unchanged with time (situation 1 and 4), it can be noticed that the real parts of the eigenvalues of matrix $\mathbf{A}$ are zero. When the norms of the relative distances between aircraft diminish with time (situation 2) the real part of the eigenvalues are negatives; finally, when those relative distances increase with time (situation 3) the real part of the eigenvalue are positive. The largest those real part are in absolute value, the more those evolutions are fast. When more aircraft are involved, a Least Mean Square regression is applied in order to extract matrix $A$ and vector $\vec{B}$. Based on the observations of aircraft (positions and speed vectors), the dynamical system has to be adjusted with the minimum error. This fitting has been done with a Least Square Minimization method. For each considered aircraft $i$, it is supposed that position $\vec{X}_{i}=$ $\left[x_{i}, y_{i}, z_{i}\right]^{T}$ and speed vector $\vec{V}_{i}=\left[v x_{i}, v y_{i}, v z_{i}\right]^{T}$ are given. An error criterion between the dynamical system model and the observations is computed :

$$
E=\sum_{i=1}^{i=N}\left\|\vec{V}_{i}-\left(A \cdot \vec{X}_{i}+\vec{B}\right)\right\|^{2}
$$

In order to use matrix forms the following matrices are introduced.

$$
\begin{gathered}
X=\left[\begin{array}{lll}
x_{1} & \ldots & x_{N} \\
y_{1} & \ldots & y_{N} \\
z_{1} & \ldots & z_{N} \\
1 & \ldots & 1
\end{array}\right] V=\left[\begin{array}{lll}
v x_{1} & \ldots & v x_{N} \\
v y_{1} & \ldots & v y_{N} \\
v z_{1} & \ldots & v z_{N}
\end{array}\right] \\
C=\left[\begin{array}{llll}
a_{11} & a_{12} & a_{13} & b_{1} \\
a_{21} & a_{22} & a_{23} & b_{2} \\
a_{31} & a_{32} & a_{33} & b_{3}
\end{array}\right]
\end{gathered}
$$

With such matrices, the error criterion $E$ can be written as : $E=\|V-C . X\|$. To minimize $E$ is the same as to minimize $E^{2}=\|V-C . X\|^{2}$. The problem is to find the matrix $C$ which minimize such a criterion. By using the matrix derivation properties, the gradient of $E^{2}$ is given by : $\nabla_{C} E^{2}=-2 .(V-C \cdot X) \cdot X^{T}$. The gradient is then canceled : 
$\nabla_{C} E^{2}=0 \Leftrightarrow C \cdot X \cdot X^{T}=V X^{T}$. The optimum matrix $C_{\text {opt }}$ is then given by $: C_{o p t}=V \cdot X^{T} \cdot\left(X \cdot X^{T}\right)^{-1}$. The expression $X^{T} .\left(X . X^{T}\right)^{-1}$ is the pseudo inverse of the matrix $X^{T}$ and can be written as : $X^{T} \cdot\left(X \cdot X^{T}\right)^{-1}=L^{T} \cdot S^{-1} \cdot R$. where $S$ is the diagonal matrix of the singular values of the matrix $X^{T}$. This singular value decomposition is very helpful in order to avoid condition troubles. The matrix $C$ is finally given by :C $=V \cdot L^{T} \cdot S^{-1} \cdot R$. The matrix $A$ is then extracted from the matrix $C$, and the associated eigenvalue decomposition is given by $A=L \cdot D \cdot U^{T}$. This first model enables to produce an aggregate metric associated to any traffic situation and can recognize any global organization pattern. It gives an aggregate measure of a given traffic situation but is not able to identify high (low) complexity areas in the airspace. For such purpose, we have developed a complexity metric based on non-linear dynamical system.

\section{Non Linear Extension In Space}

\section{A. Non linear Dynamical System}

A non linear dynamical system is summarized by the following equation :

$$
\dot{\vec{X}}(t)=\vec{f}(\vec{X})
$$

where $\vec{X}$ is the state vector of the system $\left(\vec{X}=[x, y, z]^{T}\right)$ and $\vec{f}: C^{2}$ vector field, describe systems which integral curves may fit the observed trajectories. This equation associates a vector speed $\dot{\vec{X}}$ to a position in the space coordinate $\vec{X}$ and then synthesis a particular vector field. Based on the observations of the aircraft (positions, speed vectors), the dynamical system has to be adjusted with the minimum error.This fitting is done with a Least Square Minimization (LMS) method for which the following criterion is used :

$$
E_{1}=\sum_{i=1}^{i=N}\left\|\vec{V}_{i}-\vec{f}\left(\vec{X}_{i}\right)\right\|^{2}
$$

where $N$ is number of observations.

If we consider criterion $E_{1}$ only, it can be shown that there is an infinite number of vector fields $\vec{f}$ which can be adjusted to the observations. In order to keep the smoothest one, another criterion is added which has to be minimized, the so-called "div-curl" criterion :

$$
E_{2}=\int_{R^{3}} \alpha\|\nabla \operatorname{div} \vec{f}(\vec{X})\|^{2}+\beta\|\nabla \operatorname{curl} \vec{f}(\vec{X})\|^{2} d \vec{X}
$$

with $\alpha, \beta$ positive weights controlling the smoothness of the approximation by focusing on constant divergence or constant curl. In the following, we will consider $\alpha=\beta=$ 0.5 ; in such case :

$$
E_{2}=\|\Delta \vec{f}(\vec{X})\|^{2}
$$

where $\Delta \vec{f}(\vec{X})$ is the Laplacian of the vector field $\vec{f}$.

The joint minimization of $E_{1}$ and $E_{2}$ induces a unique optimum vector field [2], [5] :

$$
\vec{f}(\vec{X})=\sum_{i=1}^{N} \phi\left(\left\|\vec{X}-\vec{X}_{i}\right\|\right) \cdot \vec{a}_{i}+\mathbf{A} \cdot \vec{X}+\vec{B}
$$

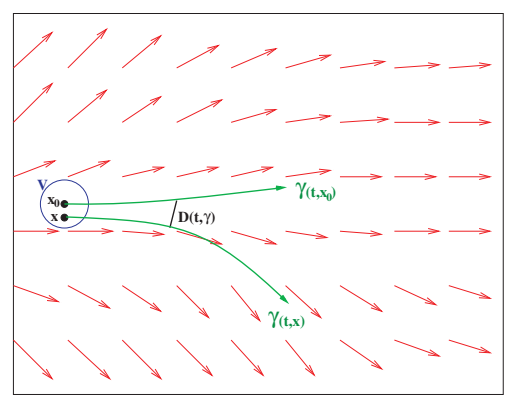

Fig. 2. Time evolution of a reference trajectory and a perturbed trajectory

with $\vec{a}_{i}$ parameter vectors (one for each observation),

$$
\phi\left(\left\|\vec{X}-\vec{X}_{i}\right\|\right)=\mathbf{Q}\left(\left\|\vec{X}-\vec{X}_{i}\right\|^{3}\right)
$$

and

$$
Q=\left[\begin{array}{lll}
\gamma & 0 & 0 \\
0 & \gamma & 0 \\
0 & 0 & \gamma
\end{array}\right]
$$

with $\gamma=\partial_{x x}^{2}+\partial_{y y}^{2}+\partial_{z z}^{2}$

The resulting adjustment is done without error $(\Rightarrow$ $\left.\min E_{1}=0\right)$.

When $\alpha=\beta=0.5$, the vector spline function $\phi$ has the following structure :

$$
\phi\left(\left\|\vec{X}-\vec{X}_{i}\right\|\right)=12 \cdot\left\|\vec{X}-\vec{X}_{i}\right\|
$$

$\left(r_{i}=\left\|\vec{X}_{i}-\vec{X}\right\|\right)$. It must be noticed, that farthest observations has more weight in such calculation. Then, in order to compute the smoothest vector field which fit exactly the measures, all observations have to be taken into account in the computation.

\section{LYAPUNOV EXPONENTS}

The metric chosen for complexity computation relies on a measure of sensitivity to initial conditions of the underlying dynamical system called Lyapunov exponents. In order to figure out what Lyapunov exponents are, let consider a point and look at its evolution when transported by the dynamical system. Let $\overrightarrow{x_{0}}$ be fixed (initial point) and let $\gamma$ be a point trajectory of the dynamical system associated to the vector field $\vec{f}$ given by :

$$
\gamma\left(t, \overrightarrow{x_{0}}\right)=\overrightarrow{x_{0}}+\int_{0}^{t} \vec{f}(u, \gamma(u, \vec{x})) d u
$$

Assume now that trajectory is disturbed by a small perturbation $\vec{\epsilon}$, we have :

$$
\gamma\left(t, \vec{x}_{0}+\vec{\epsilon}\right)=\gamma\left(t, \vec{x}_{0}\right)+\nabla_{\vec{x}} \vec{f}(\gamma(t, \vec{x})) \cdot \vec{\epsilon}+o(\|\vec{\epsilon}\|)
$$

where $\nabla_{\vec{x}} \vec{f}(t, \gamma(t, \vec{x}))$ is the differential of the vector field $\vec{f}$ at $\vec{x}$. Divergence to nominal trajectory with respect to time is thus $\left\|\gamma\left(t, \vec{x}_{0}\right)-\gamma(t, \vec{x})\right\|=D(t, s)$ (see figure 2). $\gamma(t, \vec{x})$ being defined as a flow :

$$
\frac{\partial \gamma(t, \vec{x})}{\partial t}=\vec{f}(t, \gamma(t, \vec{x})) \quad \gamma(0, \vec{x})=\vec{x}
$$


with $\vec{f}$ a smooth vector field, it is possible to show that $D(t)$ satisfies a differential equation also. Given a nominal trajectory $\gamma\left(t, \vec{x}_{0}\right)$, then divergence of nearby trajectories can be found up to order one in $\left\|\vec{x}-\vec{x}_{0}\right\|$ by solving :

$$
\frac{\partial D(t, \vec{x})}{\partial t}=\nabla_{\vec{x}} \vec{f}(t, \gamma(t, \vec{x})) \cdot D(t, \vec{x}) \quad D(0, \vec{x})=\left\|\vec{x}-\vec{x}_{0}\right\|
$$

If the three space dimensions are considered $(x, y, z)$, and since the previous equation is linear, it can be extended to the matrix form :

$$
\frac{d M(t)}{d t}=\nabla_{\vec{x}} \vec{f}(t, \gamma(t, \vec{x})) \cdot M(t) \quad M(0)=I d
$$

Where each column of the $M$ matrix corresponds to the divergence associated to the principal coordinate axis. This equation is called the variational equation of the flow. The variational equation describes in some sense a linear dynamical system "tangent" to the original one. Let $U^{t}(t) \Sigma(t) V(t)=M(t)$ be the singular value decomposition of $M(t)$. The Lyapunov exponents are mean values of the logarithms of the diagonal elements of $\Sigma(t)$ :

$$
\kappa(s)=-\frac{1}{T} \int_{0}^{T} \log \left(\Sigma_{i i}(t)\right) d t \quad \forall \Sigma_{i i}(t) \leq 1
$$

When Lyapunov exponents are high, the trajectory of a point under the action of the dynamical system is very sensitive to initial conditions (or to the parameters on which the vector field may depend), so that, situation in the future is unpredictable. On the other hand, small values of the Lyapunov exponents mean that the future is highly predictable (expected to be comfortable for a controller).

So, the Lyapunov exponent map determines the area where the underlying dynamical system is organized. It identifies the places where the relative distances between aircraft do not change with time (low real value) and the ones where such distance change a lot (high real value).

Let us now describe the practical algorithm for computing complexity maps.

\section{1) Dynamical regression based on radar observa- tions. \\ 2) The vector field and its associated gradient is then computed on each point of a cube of airspace. \\ 3) Lyapunov exponents are computed at each point of the cube by the mean of a Runge- Kutta integration.}

The figure 3 shows an example of Lyapunov exponents map for which full organized miles in trail trajectories (from south west to north east) cross two random traffic situations. This figure shows clearly a complexity valley on the miles in trail direction. This organization may have been detected even if the miles in trail trajectories would have been structured on a curve trajectory. That is the strong point of this metric: Lyapunov exponents are able to identify any kind of trajectory organization (when aircraft follow the same trajectory at the same speed).

This approach is able to produce a picture of the complexity at a given time. If we want to be able to predict the

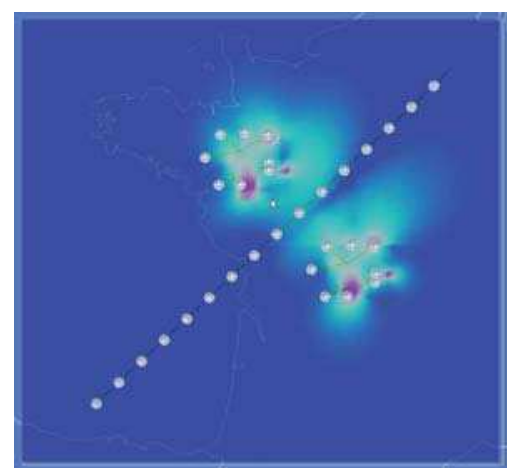

Fig. 3. Miles in trail traffic between disordered areas

complexity in a near future, one must be able to take into account time dimension in the model. This is the topic of the following section.

\section{Non Linear Extension in Space and Time}

The previous extension, is able to model air traffic at a given time but is not valid for observation with large time extension.

In such case, the vector field $\vec{f}$ has to be time dependent :

$$
\dot{\vec{X}}=\vec{f}(\vec{X}, t)
$$

As before we are looking for a vector field which both minimize the error criterion $E_{1}$

$$
\min E_{1}=\sum_{i=1}^{i=N} \sum_{k=1}^{k=K}\left\|\vec{V}_{i}\left(t_{k}\right)-\vec{f}\left(\vec{X}_{i}, t_{k}\right)\right\|^{2}
$$

and the smoothest criterion $E_{2}$ :

$$
\min E_{2}=\int_{\mathbb{R}^{3}} \int_{t}\|\Delta \vec{f}(\vec{x})\|^{2}+\left\|\frac{\partial \vec{f}}{\partial t}\right\|^{2} d \vec{x} d t
$$

The differential operator associated with this variational problem is given by :

$$
P=\frac{\partial^{2}}{\partial t^{2}}-\mu \Delta^{2}
$$

An elementary solution can be found in $\mathcal{S}^{\prime}$ as :

$$
\mathbf{f p}\left(\frac{1}{\sqrt{\mu}\|\xi\|^{2}}\right) e^{-|t| \sqrt{\mu}|\xi|^{2}}
$$

where the previous expression is the Fourier transform in $\mathcal{S}^{\prime}$, the space of rapidly decreasing smooth functions, with respect to the spatial coordinates of the elementary solution (fp stand for "Finite part" in the Hadamard sense). It turns out that this distribution is indeed obtained from a $L_{\text {loc }}^{1}$ (integrable on compact sets) mapping so that the inverse Fourier transform can be obtained readily by integration as :

$$
p(t, x)=\frac{1}{8 \pi^{3}} \int_{\mathbb{R}^{3}} \frac{1}{\sqrt{\mu}\|\xi\|^{2}} e^{\left(-|t| \sqrt{\mu}\|\xi\|^{2}\right)} e^{(i\langle x, \xi\rangle)} d \xi
$$

by Fubini's theorem and polar change of variables it becomes :

$$
p(t, x)=\frac{1}{8 \pi^{3} \sqrt{\mu}} \int_{\mathbb{R}} e^{\left(-|t| \sqrt{\mu} r^{2}\right.} \int_{\mathcal{S}^{2}} e^{(i\langle x, r s\rangle)} d \sigma(s) d r
$$


with $d \sigma$ the solid angle measure. Using a polar parametrization of the unit sphere :

$$
\begin{aligned}
& p(t, x)= \\
& \frac{1}{8 \pi^{3} \sqrt{\mu}} \int_{\mathbb{R}} e^{\left(-|t| \sqrt{\mu} r^{2}\right)} \int_{0}^{2 \pi} \int_{0}^{\pi} e^{(i\|x\| r \cos \theta)} \sin \theta d \theta d \phi d r
\end{aligned}
$$

and finally :

$$
p(t, x)=\frac{1}{2 \pi^{2} \sqrt{\mu}} \int_{\mathbb{R}} e^{\left(-|t| \sqrt{\mu} r^{2}\right)} \frac{\sin \|x\| r}{\|x\| r} d r
$$

by Parseval equality :

$$
p(t, x)=\frac{1}{4\|x\| \pi^{2}} \sqrt{\frac{\pi}{|t| \sqrt{\mu}}} \int_{-\frac{\|x\|}{2 \pi}}^{\frac{\|x\|}{2 \pi}} e^{\left(-\frac{\pi^{2} \omega^{2}}{|t| \sqrt{\mu}}\right)} d \omega
$$

so in terms of error function erf :

$$
p(t, x)=\frac{1}{4 \pi^{2}\|x\|} \operatorname{erf}\left(\frac{\|x\|}{2 \sqrt{|t| \sqrt{\mu}}}\right)
$$

The exact solution for such joint minimization problem is given by the following equation :

$\vec{f}(\vec{X}, t)=\sum_{i=1}^{N} \sum_{k=1}^{K} \psi\left(\left\|\vec{X}(t)-\vec{X}_{i}\left(t_{k}\right)\right\|,\left|t-t_{k}\right|\right) \cdot \vec{a}_{i, k}+\mathbf{A} \cdot \vec{X}+\vec{B}$

with

$$
\psi(r, t)=\operatorname{diag}\left(\frac{\sigma}{r \cdot \sqrt{\pi}} \cdot \operatorname{erf}\left[\frac{r}{\sigma} \cdot \frac{1}{\sqrt{2+\theta \cdot|t|}}\right]\right)
$$

where $\sigma$ and $\theta$ are some parameters. Furthermore, a close form of the gradient of the vector field has been identified :

$$
\frac{\partial \psi(r, t)}{\partial x}=(\alpha-\beta) \cdot x
$$

with

$$
\begin{gathered}
\alpha=\frac{2 . \sigma}{r^{2} \cdot \pi} \cdot \frac{1}{\sqrt{2+\theta \cdot|t|}} \cdot e^{-\frac{r^{2}}{\sigma^{2} \cdot(2+\theta \cdot|t|)}} \quad \beta=\frac{\psi(r, t)}{r^{2}} \\
r=\sqrt{x^{2}+y^{2}+z^{2}}
\end{gathered}
$$

The algorithm used to produce complexity map is the same as in the space extension case unless, here, the field and the associated gradient are time-dependent. As, before, one need the contribution of all aircraft in order to compute the vector field and its associated gradient at a given point. When few observations are involved $(\simeq 2000)$ the previous algorithms are tractable. When more observations are considered, one can improve the regression phase by the mean of Krylov spaces trick [6], [3] but the vector field reconstruction and gradient computation become very slow.

Another limitation of the previous approaches is linked to the robustness of the produced complexity maps. When such tools are applied to a given traffic situation, the produced maps measure the current complexity. When uncertainties has to be taken into account (uncertainties in the future position of aircraft or when we want to produce complexity map for the entire airspace in order to compare different countries (in such case flight plans are simulated with relevant noise due to the wind, flight delay, etc...)), one must use an approximation of the previous exact models for which local linear models are used.

\section{Approximation By Local Linear Models}

In order to speed up the computation of the vector field, we have developed a local approximation for which only local observations are needed for the regression. Before applying such approximation, the observations are first normalized according to the global linear model $(A \cdot \vec{X}+\vec{B})$ by subtracting the linear part to the observations :

$$
\vec{v}_{i}=\vec{V}_{i}-\left(A \cdot \vec{X}_{i}+\vec{B}\right)
$$

Then, such approximation will locally update the linear regression model in order to fit observations.

The first order space-time linear expansion of a vector field $\vec{f}(t, \vec{X})$ is given by the following expression :

$$
\begin{gathered}
\vec{f}\left(t_{0}, \vec{X}_{0}\right)=\vec{f}(t, \vec{X})+\frac{\partial \vec{f}(t, \vec{X})}{\partial t}\left(t_{0}-t\right)+ \\
+\frac{\partial \vec{f}(t, \vec{X})}{\partial \vec{X}}\left(\vec{X}_{0}-\vec{X}\right)+O\left(\left|t_{0}-t\right|+\left\|\vec{X}_{0}-\vec{X}\right\|\right)
\end{gathered}
$$

where $\frac{\partial \vec{f}(t, \vec{X})}{\partial t}$ is the time derivative vector of the vector field $f$ and $+\frac{\partial \vec{f}(t, \vec{X})}{\partial \vec{X}}$ is the associated space derivatives matrix. This equation represents a local linear model of the vector field $\vec{f}$ in the vicinity of $(t, \vec{X})$. This approximation is only valid near a given point $(t, \vec{X})$. We propose to use this model in order to compute the vector field base on a given set of local observations. First, a point $(t, \vec{X})$ in the state space is considered (a grid point) for which observations are searched in its vicinity. The field at this point $(\vec{f}(t, \vec{X}))$ is then computed in order to minimize the errors between local observations $\vec{v}_{i}\left(t_{i}, \vec{X}_{i}\right)$ and the linear expansion of the vector field $\vec{f}$ at the grid point $(t, \vec{X})$

$$
\begin{gathered}
\vec{v}_{i}\left(t_{i}, \vec{X}_{i}\right) \simeq \vec{f}(t, \vec{X})+\frac{\partial \vec{f}(t, \vec{X})}{\partial t}\left(t_{i}-t\right)+\frac{\partial \vec{f}(t, \vec{X})}{\partial \vec{X}}\left(\vec{X}_{i}-\vec{X}\right) \\
=\vec{a}+\vec{b} \cdot\left(t_{i}-t\right)+C \cdot\left(\vec{X}_{i}-\vec{X}\right)
\end{gathered}
$$

where $\vec{a}=\vec{f}(t, \vec{X}), \vec{b}=\frac{\partial \vec{f}(t, \vec{X})}{\partial t}$ and $[C]=\frac{\partial \vec{f}(t, \vec{X})}{\partial \vec{X}}$

We are seeking vectors $\vec{a}, \vec{b}$ and matrix $[C]$ which minimize the error criterion $J$ :

$$
\begin{gathered}
\min _{\vec{a}, \vec{b}, C} J= \\
\sum_{i=1}^{N}\left\|\vec{v}_{i}\left(\vec{X}_{i}, t_{i}\right)-\left\{\vec{a}+\vec{b}\left(t_{i}-t\right)+C \cdot\left(\vec{X}_{i}-\vec{X}\right)\right\}\right\|^{2} \cdot \psi_{i}
\end{gathered}
$$

where $\psi_{i}=\psi\left(t_{i}-t, \vec{X}_{i}-\vec{X}\right)$ is a space-time weight function which select the closest observations. If we note

$$
\begin{gathered}
X=\left[\begin{array}{ccc}
1 & \ldots & 1 \\
\left(t_{1}-t\right) & \ldots & \left(t_{N}-t\right) \\
\left(x_{1}-x\right) & \ldots & \left(x_{N}-x\right) \\
\left(y_{1}-y\right) & \ldots & \left(y_{N}-y\right) \\
\left(z_{1}-z\right) & \ldots & \left(z_{N}-z\right)
\end{array}\right] \\
V=\left[\begin{array}{ccc}
v x_{1} & \ldots & v x_{N} \\
v y_{1} & \ldots & v y_{N} \\
v z_{1} & \ldots & v z_{N}
\end{array}\right] \Psi=\left[\begin{array}{cccc}
\psi_{1} & \ldots & 0 \\
\ldots & \ldots & \ldots \\
0 & \ldots & \psi_{N}
\end{array}\right] \\
M=\left[\begin{array}{ccccc}
a_{x} & b_{x} & C_{x x} & C_{x y} & C_{x z} \\
a_{y} & b_{y} & C_{y x} & C_{y y} & C_{y z} \\
a_{z} & b_{z} & C_{z x} & C_{z y} & C_{z z}
\end{array}\right]
\end{gathered}
$$


The criterion $J$ can be written as :

$$
J=\| \Psi\left(M . X-V \|^{2}=(M . X-V)^{T} . \Psi \cdot(M . X-V)\right.
$$

By differentiating $J$ with respect to $M$, we find :

$$
M_{o p t}=V \cdot \Psi \cdot X^{T}\left(X \cdot \Psi X^{T}\right)^{-1}
$$

In order to avoid condition trouble, a singular value decomposition will be used to compute such matrix. We then propose the following algorithm to compute complexity maps :

1) Global linear regression $(A \cdot \vec{X}+\vec{B})$

2) Computation of the relative observations $\left(\vec{v}_{i}-\right.$ $(A \cdot \vec{X}+\vec{B}))$.

3) For each grid point $\vec{X}(t)$

a) Compute the Local Linear Model $(\vec{a}, \vec{b}, C)$

b) Vector field computation $((A \cdot \vec{X}+\vec{B})+\vec{b})$

c) Gradient matrix computation $A+C$

4) Lyapunov exponents computation by time integration

Step 3 and 4 can be done in parallel in order to speed up the computation.

\section{A. Stochastic extension}

When countries have to be compared in term of airspace, one must consider many days of traffic with regular flight plans for which observations are sample of a random process. In this framework, positions of aircraft on their trajectories undergo random deviations due to wind, delay, etc ... Even when one want to predict complexity in a given area, one must be able to take into account uncertainties of future positions. For both applications, time shift random process is the most relevant model, for which the time of observation $t_{i}$ is replaced by $t_{i}-\tilde{\tau}_{i}$, where $\tilde{\tau}_{i}$ is a random variables (all random variable will be note with a upper tilde .).

Then $t_{i}$ is now a random variable : $\tilde{t}_{i}=t_{i}-\tilde{\tau}_{i}$ We have $\tilde{\vec{X}}_{i}=\vec{\gamma}\left(\tilde{t}_{i}\right), \tilde{\vec{v}}_{i}=\dot{\vec{\gamma}}\left(\tilde{t}_{i}\right)-A \cdot \tilde{\vec{X}}_{i}+\vec{B}$ and $\tilde{\psi}_{i}=\psi\left(\tilde{t}_{i}-t, \tilde{\vec{X}}_{i}-\right.$ $\vec{X})$, where $\vec{\gamma}(t)$ is the aircraft trajectory. The criterion $J$ becomes a random variable and we are seeking $\vec{a}, \vec{b}, C$ in order to minimize the expected value of $J$ :

$$
\begin{gathered}
\min _{\vec{a}, \vec{b}, C} E[\tilde{J}]= \\
\sum_{i=1}^{N} E\left[\left\|\tilde{\vec{v}}_{i}-\left[\vec{a}+\vec{b} \cdot\left(\tilde{t}_{i}-t\right)+C \cdot\left(\tilde{\vec{X}}_{i}-\vec{X}\right)\right]\right\|^{2} \cdot \tilde{\psi}_{i}\right]
\end{gathered}
$$

If we note $E[\tilde{V}], E[\tilde{X}]$ and $E[\tilde{\Psi}]$ the expected value of the random matrices $\tilde{V}, \tilde{X}, \tilde{\Psi}$ the criterion $E[\tilde{J}]$ can be written as :

$$
\begin{gathered}
E[\tilde{J}]=\|E[\tilde{\Psi}](M \cdot E[\tilde{X}]-E[\tilde{V}])\|^{2} \\
=(M \cdot E[\tilde{X}]-E[\tilde{V}])^{T} \cdot E[\tilde{\Psi}] .(M \cdot E[\tilde{X}]-E[\tilde{V}])
\end{gathered}
$$

By differentiating $E[\tilde{J}]$ with respect to $M$, we find :

$$
M_{o p t}=E[\tilde{V}] \cdot E[\tilde{\Psi}] \cdot E[\tilde{X}]^{T}\left(E[\tilde{X}] \cdot E[\tilde{\Psi}] E[\tilde{X}]^{T}\right)^{-1}
$$

The same algorithm as the one propose in the deterministic case will be used for computing complexity maps.

\section{CONCLUSION}

We have presented in this paper a new air traffic complexity metric based on vector field model of air traffic. Most of the work has been devoted to improvements in interpolating splines used to fit a vector field to the observation. Unlike linear models which produce mean complexity indicators, the non-linear one may give local information, thus providing a way of displaying maps of complexity. A local approximation has also been proposed in order to parallelize the computation and to improve the robustness of the map produced when time shift noise is included in observations.

In a future work, such a tool will be applied to comparison of US and Europe airspace by producing complexity map of USA and Europe for a full day of traffic.

\section{REFERENCES}

[1] G Aigoin, Air traffic complexity modeling, Master's thesis, Ecole Nationale de l'Aviation Civile, 2001

[2] L Amodei and M.N Benbourhim, A vector spline approximation, Journal of Approximation Theory 67 (1991), 51-79.

[3] Ake Bjorck, Numerical methods for least squares problems, Society for Industrial \& Applied Mathematics, 1996.

[4] D Delahaye and S Puechmorel, Air traffic complexity : Towards intrinsic metrics, Proceedings of the Second USA/EUROPE ATM R\&D Seminar, Eurocontrol/FAA, 2000.

[5] D. Suter F. Chen, Fast evaluation of vector splines in three dimensions, Computing.

[6] M.H. Gutknecht, A brief introduction to krylov space methods for solving linear systems, Proceedings of the International Symposium on Frontiers of Computational Science, 2005.

[7] B Hilburn, Cognitive complexity and air traffic control: A literature review, Tech. report, Eurocontrol, 2004

[8] J.M Histon, G Aigoin, D Delahaye, R.J Hansman, and Puechmorel. $\mathrm{S}$, Introducing structural consideration into complexity metrics, Proceedings of the Fourth USA/EUROPE ATM R\&D Seminar, Eurocontrol/FAA, 2001.

[9] J.M Histon, R.J Hansman, G Gottlieb, H Kleinwaks, S Yenson, D Delahaye, and Puechmorel. S, Structural consideration and cognitive complexity in air traffic control, Proceedings of the $21^{\text {th }}$ Air Traffic Management for Commercial and Military Systems, IEEE,AIAA, 2002.

[10] Windemere inc, An evaluation of air traffic control complexity, Tech. report, NASA 2-14284, 1996.

[11] B Kirwan, R Scaife, and R Kennedy, Investigating complexity factors in u.k. air traffic management, Human Factors and Aerospace Safety 1(2) (2001).

[12] I.V Laudeman, S.G Shelden, R Branstrom, and C.L Brasil, Dynamic density : an air traffic management metric, Tech. report, NASA TM1998-112226, 1998.

[13] R.H Mogford, J.A Guttman, P Morrowand, and P Kopardekar, The complexity construct in air traffic control : A review and synthesis of the literature, Tech. report, FAA, 1995.

[14] RTCA, Atfcm and capacity report, Tech. report, Eurocontrol, 2006.

[15] B Sridhar, K.S Seth, and S Grabbe, Airspace complexity and its application in air traffic management, Proceedings of the Second USA/EUROPE ATM R\&D Seminar, Eurocontrol/FAA, 2001. 